\title{
ESTUDIOS DE SUEÑO EN PACIENTES CON ENFERMEDADES NEUROMUSCULARES
}

\section{SLEEP BREATHING DISORDERS IN CHILDREN WITH NEUROMUSCULAR DISEASES}

\author{
Daniel Zenteno $\mathrm{A}^{(1)}$, Daniela Verbal $\mathrm{C}^{(2)}$, Camilo Barraza $\mathrm{E}^{(2)}$, María José Elso $\mathrm{T}^{(3)}$ \\ 1. Pediatra Broncopulmonar, Hospital Guillermo Grant Benavente, Concepción. \\ Profesor Asistente, Departamento de Pediatría, Universidad de Concepción. \\ 2. Interno Medicina, Universidad de Concepción. \\ 3. Neurólogo, Hospital Guillermo Grant Benavente, Concepción. \\ Profesor Colaborador, Departamento de Neurología, Universidad de Concepción.
}

\begin{abstract}
Sleep disordered breathing (SDB) is frequent in pediatric patients with neuromuscular diseases, generated by different pathophysiological mechanisms that usually lead to hypoventilation and obstructive sleep apnea hypopnea syndrome. Diagnostic testing should be conducted early and with a preset frequency, even more if there is the clinical suspicion of SDB and respiratory function impairment. There are different diagnostic studies, each with its advantages and disadvantages. Polysomnography is considered the reference standard and alternatively, polygraphy has shown a high level of correlation with respiratory events.
\end{abstract}

Key words: neuromuscular diseases, respiratory sleep disorders, hypoventilation, polysomnography

RESUMEN

Los trastornos respiratorios del sueño (TRS) son frecuentes en los pacientes pediátricos con enfermedades neuromusculares, siendo generados por diversos mecanismos fisiopatológicos que usualmente llevan a hipoventilación y síndrome de apnea hipopnea obstructiva del sueño. La indicación de estudio debe ser precoz y con una periodicidad prestablecida, más aun frente a la sospecha clínica de TRS y al constatar alteraciones funcionales respiratorias. Existen distintos estudios para su diagnóstico y seguimiento, incluso en pacientes con soporte ventilatorio no invasivo; cada uno con ventajas e inconvenientes particulares. Destaca la polisomnografía, como estándar de referencia y como alternativa, la poligrafía que ha demostrado tener un alto índice de correlación con eventos respiratorios.

Palabras clave: enfermedad neuromuscular, trastornos respiratorios del sueño, hipoventilación, polisomnografía

\section{INTRODUCCIÓN}

Los pacientes con enfermedades neuromusculares (ENM) pueden manifestar trastornos respiratorios del sueño (TRS) en distintas etapas de su evolución. Se ha reportado una prevalencia sobre el $40 \%$ de TRS en este grupo de pacientes, en algún momento de sus vidas; tendrían un riesgo 20 veces mayor que la población pediátrica general ${ }^{(1)}$. En nuestro país, Brockmann et al publicaron su experiencia en pacientes con ENM candidatos a ventilación mecánica no invasiva (AVNI), en los cuales en un $71 \%$ se constató algún TRS ${ }^{(2)}$.

En consideración a la mayor prevalencia, mayor cantidad y calidad de evidencia en el tema, la distrofia muscular de Duchenne (DMD) es considerada enfermedad prototipo de las ENM de progresión lenta. Otras frecuentemente asociadas a TRS son: Atrofia Espinal I, II y III, distintos tipos de distrofias musculares, distrofias miotónicas y escapulohumerales.

\section{Correspondencia:}

Dr Daniel Zenteno Araos

Avenida Alemana 98, Pedro de Valdivia, Concepción, Chile

Correo electrónico danielzenteno@gmail.com 


\section{FISIOPATOLOGÍA}

Como mecanismos fisiopatológicos de los TRS en estos pacientes, se encuentran: alteraciones del control ventilatorio, de la quimiosensibilidad y de los músculos respiratorios, especialmente el diafragma, a lo cual se suma a una mayor resistencia de la vía aérea superior y la afección del clearence mucociliar. Estas alteraciones poseen un carácter progresivo; en etapas iniciales, se encuentran especialmente presentes en etapa del sueño REM, lo cual puede predisponer a la aparición de hipoventilación, frecuentemente asociada a síndrome de apnea hipopnea obstructiva del sueño (SAHOS). Por otro lado el mecanismo fisiopatológico que determinará el TRS depende de la enfermedad de base. Por ejemplo en los pacientes con atrofia muscular espinal y enfermedad de Duchenne, en las cuales el compromiso muscular es generalizado, habrá apneas obstructivas e hipoventilación. En la distrofia miotónica, en la cual hay compromiso de la musculatura de vía aérea superior, diafragma y hay alteración del drive ventilatorio además aparecen apneas centrales. (3)

\section{ESTUDIO DE LOS TRS}

Entre las herramientas para el estudio del sueño en estos pacientes la polisomnografía (PSG) también es considerada como gold standard para el diagnóstico de los TRS. Además se cuenta con la poligrafía (PG), alternativa abreviada aplicable a población pediátrica, y la saturometría nocturna continua, que posee menor grado de precisión. La evaluación de la fuerza muscular respiratoria mediante registro de presiones estáticas (presión inspiratoria máxima (Pimáx) o sniff nasal, presión espiratoria máxima (Pemáx), medición de capacidad vital forzada (CVF) con espirometría ayudan a predecir la presencia de TRS. La gasometría y la capnometría también forman parte de la evaluación de estos pacientes $(4,5)$ (Figura 1)

Figura 1. Exámenes utilizados para el estudio de los TRS en enfermedades neuromusculares.

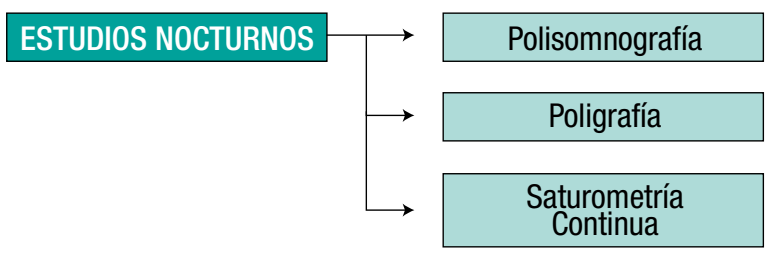

\section{ESTUDIOS DIURNOS}

Fza Muscular Resp

Espirometría

Gases en sangre

Capnometría

\section{INDICACIONES}

Un paciente con ENM tiene indicación de estudio de sueño siempre que se sospeche un SAHOS o hipoventilación, si presenta una debilidad muscular progresiva o en el caso de los pacientes con enfermedad de Duchenne cuando inicia la pérdida de la deambulación. También debe estudiarse en pacientes que cuenten con estudio funcional respiratorio que evidencie una CVF disminuida 0 hay una debilidad muscular inspiratoria (6) (Tabla 1)

Tabla1. Indicación para estudios de sueño en pacientes con enfermedades neuromusculares.

\begin{tabular}{|c|c|}
\hline Indicaciones & Observaciones \\
\hline $\begin{array}{c}\text { Síntomas SAHOS y/0 } \\
\text { hipoventilación }\end{array}$ & Ronquido, apnea, cefalea matinal \\
\hline $\begin{array}{c}\text { Pérdida ambulación (debilidad } \\
\text { progresiva o nunca han caminado) }\end{array}$ & $\begin{array}{c}\text { Incapacidad de caminar asociada } \\
\text { moderada-severa debilidad muscular }\end{array}$ \\
\hline CVF $<60 \%$ & Más confiable en Z $>6$ años \\
\hline Debilidad diafragmática & Puede confirmarse con Pimax 0 sniff \\
\hline
\end{tabular}

SAHOS: síndrome apnea hipopnea obstructiva del sueño, CVF: capacidad vital forzada, TRS: trastorno respiratorio del sueño, Pimax: presión inspiratoria máxima.

En el caso de los pacientes con DMD, los consensos internacionales sugieren que deben ser derivados a pediatra broncopulmonar lo más precoz posible para su seguimiento; se recomienda realizar estudios del sueño a los 5 años 0 antes si existe clínica de SAHOS o hipoventilación, en caso de pérdida de bipedestación 0 si poseen alguna alteración funcional respiratoria $(7,8)$.

\section{ESTUDIOS DURANTE EL SUEÑO}

Al realizar una PSG se recomienda incorporar la capnometría en los registro de estos pacientes y si procede, adicionar canales necesarios para iniciar el soporte ventilatorio $(6,9)$.

Las hipopneas son el hallazgo más precoz y frecuente. Algunos autores describen los eventos de hipopneas pseudocentrales, en las cuales hay una reducción del flujo respiratorio relacionado con una disminución de los movimientos torácicos, con mantención de movimientos abdominales y desaturaciones $\geq 3 \%$; más que un evento obstructivo propiamente tal, se estima que sería secundaria a debilidad inicial de la musculatura inspiratoria $(3,10)$ 
Figura 2. Eventos pseudocentrales.

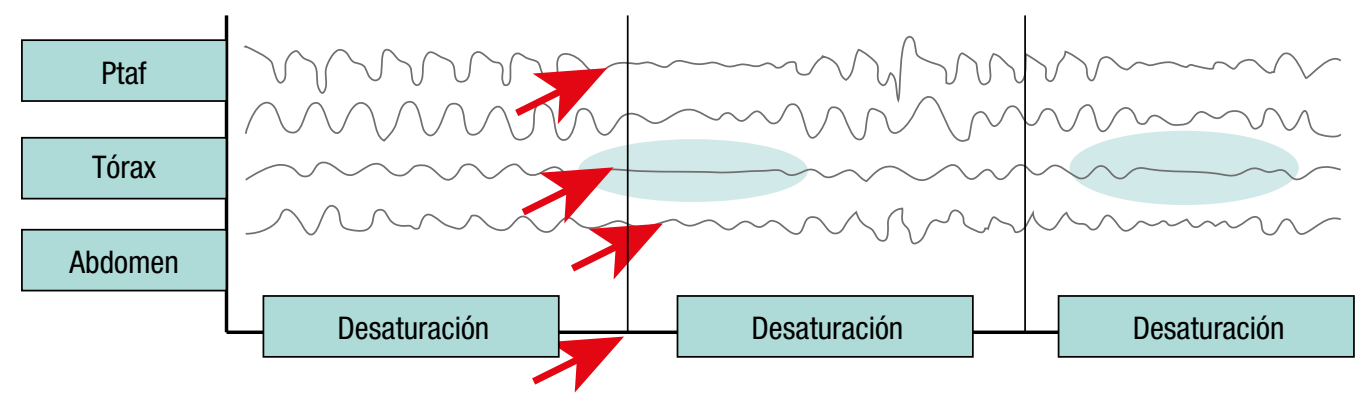

Ptaf: sensor de flujo por presión

Se aprecia una reducción del flujo con ptaf, disminución de la curva torácica con mantención de la abdominal, y desaturación (flechas).

Existen distintas definiciones para hipoventilación, siendo una de las más utilizadas una PaC02 mayor a $50 \mathrm{mmHg}$ en más de un $25 \%$ del tiempo de registro validado. Algunos autores proponen puntos de corte más bajos. La hipoventilación se constata inicialmente en el sueño REM. El compromiso es progresivo, surgiendo apneas obstructivas, y en una etapa más avanzada cuando existe compromiso bulbar, se presentan apneas centrales ${ }^{(3)}$

La PG nos permite una evaluación cardiorrespiratoria, sin incluir parámetros neurofisiológicos; ha demostrado en varios estudios que posee un índice de correlación alto con la polisomnografía en relación a eventos e índices respiratorios. Se usa como método diagnóstico y para seguimiento en las ENM, y para control del apoyo ventiatorio ${ }^{(6,11,12)}$.

\section{ESTUDIOS PREDICTORES DIURNOS}

Las alteraciones encontradas en la función muscular respiratoria se relacionan con la presencia de TRS, pero su alteración por lo general ocurre en una etapa avanzada de la enfermedad ${ }^{(4,5,13)}$. La espirometría está ampliamente disponible. Cuando existe una CVF menor del 60\% del valor teórico podría ya existir algún TRS. Se ha visto hipoventilación significativa cuando su valor es inferior al $40 \%$ y existen síntomas diurnos con menos de $25 \%$. . $^{(4)}$

La fuerza muscular inspiratoria puede ser evaluada con Pimáx, el parámetro que más se ha estudiado en relación a TRS $(15,16)$. Además de relacionarse con la presencia de un TRS permite establecer estrategias de rehabilitación respiratoria. Valores de Pimax menores de $45 \mathrm{cmH} 20$ se constatan al inicio de los TRS, cuando es menor de $35 \mathrm{cmH} 20$ ya hay síntomas respiratorios severos. La gasometríatambién es un examen básico que puede estar disponible en la mayoría de los hospitales de nuestro medio, pero que habitualmente se altera en etapas avanzadas de la enfermedad. La muestra debe obtenerse al momento de despertar ${ }^{(5,13)}$.

El C02 puede medirse en el gas exhalado (ETC02) 0 transcutáneo (TCPC02). En el primer casose puede medir usando distintas interfaces (nasales, nasobucales, traqueostomía, etc.
La TCPCO2 puede ser medida durante un registro aislado o en el registro continuo de un estudio de sueño, pudiendo incluirse al registro simultaneo de otros canales y permitiendo ver las variaciones asociadas a eventos respiratorios. La tecnología Sentec, permite el registro de TcPCO2, ha sido estudiada en pacientes pediátricos con soporte ventilatorio invasivo y no invasivo, durante su seguimiento y proceso de destete, asociados a poligrafía y actimetria. ${ }^{(17,18)}$

\section{DIAGNÓSTICO DE LOS TRS}

Los pacientes con ENM pueden debutar precozmente con un trastorno significativo del comando ventilatorio, que los lleve rápidamente a la intubación y a la traqueostomía. Sin embargo, la mayoría se presentan en el contexto de una enfermedad lentamente progresiva, cuya evolución natural implica un deterioro progresivo, por lo tanto, es importante su estudio precoz para asumir conductas específicas, como el inicio de la ventilación mecánica no invasiva.

Aproximadamente a los 10 años de edad, los niños con DMD, comienzan a perder su capacidad funcional respiratoria, situación que puede identificarse en forma temprana por medio de estudios de sueño, por lo general en la etapa de sueño REM ${ }^{(19)}$ Nozoe et al evaluaron pacientes sintomáticos con DMD versus controles de una edad similar, donde claramente se evidencian en el grupo con DMD presentaba una alteración en la eficiencia del sueño, de latencias de inicio y porcentaje de sueño REM, más hipopneas, más apneas obstructivas y mayores índices respiratorios, predominantemente en etapa del sueño $\mathrm{REM}^{(20)}$

Nuestro grupo publicó recientemente una experiencia sobre el diagnóstico de los TRS en pacientes con ENM, fundamentalmente con antecedentes de DMD, derivados a un policlínico de especialidad. Se realizaron $31 \mathrm{PG}$ en niños hospitalizados con ENM, donde las PG validadas correspondieron al $88 \%$, similar a otras experiencias pediátricas. Destacó la determinación de conducta, luego de haber realizado la PG; en el $66 \%$ de los pacientes la conducta fue iniciar la ventilación mecánica no invasiva. Sin embargo, no encontramos relación diferencias entre la severidad del SAHOS y la conducta asumida. Esto se debe probablemente, a que la indicación de soporte ventilatorio, no se basa solamente en un estudio del sueño; existen una serie de otros condicionantes: clínicos, estudios complementarios, aspectos sociales y vinculados con la adherencia ${ }^{(12)}$. 


\section{SEGUIMIENTO DE LOS TRS}

Luego de confirmar un TRS, y en caso de existir alteración que sugiera el inicio de AVNI, puede considerarse establecer los parámetros ventilatorios mediante PSG o PG.

Existen recomendaciones internacionales, las cuales establecen algoritmos para la titulación de los pacientes con SAHOS; también existen sugerencias para la titulación de pacientes con síndromes de hipoventilación. Se sugiere titular a estos pacientes con presión continua en la vía aérea ( CPAP) o con presión de soporte binivelada (BIPAP) considerando como principal parámetro, la presión de soporte, la cual proporciona estabilidad a la vía aérea y ventilación adecuada se puede iniciar con presión inspiratoria ((Ipap) de 8 y espiratoria (Epap) de $4 \mathrm{~cm}$ H20, con sus máximas según edad del paciente: Ipap máximo de 20 para menores de 12 años y de $30 \mathrm{~cm} \mathrm{H2O} \mathrm{para} \mathrm{mayores.}$ La presión de soporte mínima es de $4 \mathrm{~cm} \mathrm{H} 20$, pero puede ser mayor según los requerimientos del paciente. Según los resultados obtenidos, se aumenta cada 5 minutos las presiones del ventilador, considerando un aumento de la presión de soporte de $102 \mathrm{~cm} \mathrm{H} 20$, en cada instancia. ${ }^{(21,22)}$

En pacientes con una ENM más avanzada, se pueden constatar eventos centrales para lo que se debe adicionar una frecuencia respiratoria de respaldo. Lo que se recomienda es iniciar con una frecuencia de al menos 10 por minuto y aumentar según los resultados. En caso necesario también se puede ajustar el sporte adicional de oxigeno; lo que se recomienda cuando hay saturaciones en vigilia menores de $88 \%$ y en pacientes que emplean Bipap asociado a frecuencia respiratoria de respaldo, en caso que posean parámetros ventilatorios altos y persisten con una saturación menor a $90 \%$ por más de 5 minutos. ${ }^{(21,22)}$

La terapia ventilatoria puede evaluarse con estudios de sueño, PSG y PG, además de capnometría y actimetría. ${ }^{(23-25)}$

La recomendación de las guías británicas y americanas en paciente con DMD, es que el control de estudios de sueño, sea anual en un paciente que no tenga alteración en los exámenes previos y en los pacientes con AVNI; ambas recomiendan realizar estas evaluaciones, en los pacientes en que surgen síntomas sugerentes de SAHOS o poseen una evolución respiratoria tórpida. ${ }^{(6,7)}$

Interesantemente, un grupo europeo de especialistas en sueño y ventilación mecánica, establece ciertos eventos, además de los convencionales, que pudiesen tener repercusión en el sueño, gasométrica, adherencia al tratamiento y potencialmente en los resultados terapéuticos ${ }^{(26)}$. Ellos definen los escapes involuntarios, las asincronías con la ventilación mecánica, la disminución del comando respiratorio, la obstrucción de la vía aérea superior con 0 sin alteración del comando respiratorio y los eventos mixtos ${ }^{(24)}$. Es un tema bastante complejo que probablemente tendrá más desarrollo en los próximos años; aun esta pendiente determinar cuáles serán los puntos de corte para establecer alteración y la automatización inicial de este tipo de reportes. La principal relevancia de esta propuesta, es que a partir de estas alteraciones, se establecen ciertas alternativas de solución ${ }^{(10)}$. Por ejemplo, si hay escapes significativamente elevados, se podría establecer un cambio de mascarilla o revisar la fijación de la interface (Tabla 2).
Tabla 2. Características, causas y alternativas de solución de eventos respiratorios relacionados con la asistencia ventilatoria no invasiva.

\begin{tabular}{|c|c|c|c|}
\hline $\begin{array}{l}\text { Trastorno } \\
\text { sueño } \\
\end{array}$ & Causa & PSG características & \begin{tabular}{|l|} 
Solución / \\
Alternativas \\
\end{tabular} \\
\hline Escape aéreo & $\begin{array}{l}\text { Ajuste máscara, } \\
\text { PVA }\end{array}$ & $\begin{array}{l}\text { Caída señal PVA, ampu- } \\
\text { tación F espiratorio, } \uparrow T i \text {, } \\
\downarrow \text { señal TA }\end{array}$ & $\begin{array}{l}\text { Cambio máscara, } \\
\text { considerar fijación } \\
\text { mentón }\end{array}$ \\
\hline $\begin{array}{l}\text { Respiración } \\
\text { inefectiva }\end{array}$ & $\begin{array}{l}\text { Debilidad muscular, } \\
\text { hiperinflación } \\
\text { dinñamica }\end{array}$ & $\begin{array}{l}\text { Alta presión, esfuerzos } \\
\text { en flujo, TCA y señales } \\
\text { no relacionados presión } \\
\text { de equipo. Rem más }\end{array}$ & $\begin{array}{l}\downarrow \mathrm{PS}, \uparrow \text { sensibilidad } \\
\text { trigger, } \uparrow \text { Epap }\end{array}$ \\
\hline Autotrigger & $\begin{array}{l}\text { Escape, conden- } \\
\text { sación, variabilidad } \\
\text { cardíaca }\end{array}$ & $\begin{array}{l}\text { Más de } 3 \text { eventos } \\
\text { sucesivos de entrega } \\
\text { excesiva de FR del pte. } \\
\text { Mas N Remo N1 y N2 }\end{array}$ & $\begin{array}{l}\text { Ajustar máscara, } \\
\downarrow \text { trigger, drenar } \\
\text { circuito }\end{array}$ \\
\hline $\begin{array}{l}\text { Insuflación } \\
\text { prolongada }\end{array}$ & $\begin{array}{l}\text { Escape, dispositivos } \\
\text { de volumen } \\
\text { asegurado }\end{array}$ & Más en NRem, N2 & $\begin{array}{l}\text { Corregir escapes, } \\
\text { acrotar } \mathrm{Ti}\end{array}$ \\
\hline Apnea Central & $\begin{array}{l}\text { Ventialción de CO2 } \\
\text { bajo el umbral hipo- } \\
\text { cápnico apneico }\end{array}$ & $\begin{array}{l}\text { Ausencia de flujo y } \\
\text { esfuerzo TCA, asociado a } \\
\downarrow \text { CO2. No en Rem }\end{array}$ & $\downarrow P S$ \\
\hline Cierre glótico & $\begin{array}{l}\text { Exceso soporte } \\
\text { ventilatorio/ } \\
\text { hiperventilación }\end{array}$ & $\begin{array}{l}\text { Ausencia o } \downarrow \text { excursión } \\
\text { TA con respiración por } \\
\text { equipo. Más en N2, } \\
\text { asociada a hiperventila- } \\
\text { ción y C02 bajo }\end{array}$ & $\begin{array}{l}\downarrow \text { FR y(o PS, } \uparrow \text { Epap, } \\
\text { uso máscara nasal, } \\
\uparrow \text { espacio muerto }\end{array}$ \\
\hline
\end{tabular}

Fauroux et al evaluaron 39 menores con AVNI; aplicando poligrafía, capnometría y actimetría simultáneas, detectando eventos respiratorios asociados a desaturaciones 0 a despertares. Posteriormente, establecieron estrategias para el ajuste de los parámetros ventilatorios según los resultados obtenidos y repitieron estudios iniciales logrando una disminución de los índices respiratorios. Los fenómenos que más se relacionaron a los eventos fueron las asincronías con el ventilador y los escapes, que dan cuenta de un 33 y $27 \%$ de los eventos detectados respectivamente. Por otro lado, los escapes fueron los que más se relacionaron a alteraciones en la actimetría. $^{(27)}$

Algo menos estudiado, pero que puede realizarse en centros especializados, es evaluar con estudio de sueño a pacientes con ventilación invasiva prolongada. En estos casos se puede ocupar un sensor de flujo a la traqueostomía; puede ayudar a la determinación del modo ventilatorio, a descartar 0 detectar eventos respiratorios y la evaluación a pacientes de destete del ventilador.

En los procesos de decanulación hay diversas estrategias que pueden emplearse, como la evaluación funcional respiratoria y el entrenamiento muscular respiratorio en pacientes cooperadores. Un estudio de sueño antes y después de la decanulación, podría ser útil para determinar la estrategia a utilizar posteriormente. Fauroux et al publicaron un artículo sobre decanulación programada; realizaron $P G$ precoces post decanulación, estableciendo el efecto positivo de la ventilación mecánica no invasiva en esta etapa. ${ }^{(27)}$ 


\section{CONCLUSIÓN}

En pacientes con ENM, existe una variedad de herramientas diagnósticas que pueden ser complementarias entre ellas. El diagnóstico de los TRS en este grupo de pacientes es relevante y una intervención precoz puede generar repercusiones favorables que condicionen su morbimortalidad. El seguimiento con estudios de sueño es recomendable, ya sea con o sin ventilación mecánica, dependiendo de la condición de cada paciente y con una periodicidad prestablecida.

\section{Los autores declaran no presentar conflicto de interés}

\section{REFERENCIAS}

1. Gozal D. Pulmonary manifestations of neuromuscular disease with special reference to Duchenne muscular dystrophy and spinal muscular atrophy. Pediatr Pulmonol 2000; 29: 141-50.

2. Brockmann V P, Holmgren $P N$, Salinas M P, Prado A F. Utilidad de un cuestionario clínico para el diagnóstico de trastornos respiratorios del sueño en niños con enfermedades neuromusculares. Rev Chil Pediatr 2011; 82: 319-28.

3. Arens R, Muzumdar H. Sleep, sleep disordered breathing, and nocturnal hypoventilation in children with neuromuscular diseases. Paediatr Respir Rev. 2010 ;11(1):24-30.

4. Zenteno A D, Salinas F P, Vera U R, Brockmann V P, Prado A F. Enfoque Pediátrico para el Estudio de los Trastornos Respiratorios del Sueño. Rev Chil Pediatr 2010; 81: 445-55.

5. Katz S L. Assessment of sleep-disordered breathing in pediatric neuromuscular diseases. Pediatrics 2009; 123 Suppl 4: $222-5$

6. Hull J, Aniapravan R, Chan E, Chatwin M, Forton J, Gallagher J, Gibson N, Gordon J, Hughes I, McCulloch R, Russell RR, Simonds A. British Thoracic Society guideline for respiratory management of children with neuromuscular weakness. Thorax 2012;67:1-40.

7. Alves RS, Resende MB, Skomro RP, Souza FJ, Reed UC. Sleep and neuromuscular disorders in children. Sleep Med Rev. 2009 ;13:133-48.

8. Finder J D, Birnkrant D, Carl J, Farber H J, Gozal D, lannaccone $\mathrm{S} T$, et al. Respiratory care of the patient with Duchenne muscular dystrophy: ATS consensus statement. Am J Respir Crit Care Med 2004; 170: 456-65.

9. Standards and indications for cardiopulmonary sleep studies in children. American Thoracic Society. Am J Respir Crit Care Med. 1996;153:866-78.

10. Aboussouan LS. Sleep-disordered Breathing in Neuromuscular Disease. Am J Respir Crit Care Med. 2015 1;191:979-89.

11. Alonso M L, Teran S J, Cordero J A, Navazo A I, Ordax E, Masa $J F$, et al. Reliability of respiratory polygraphy for the diagnosis of sleep apneahypopnea syndrome in children. Arch Bronconeumol 2008; 44: 318-23.

12. Zenteno D, Rodríguez I, Rivas C, Peña R, Molina I, Tapia J. Poligrafía en niños con enfermedad neuromuscular. Rev Chil Enferm Respir 2015; 31: 152-159.

13. Hukins CA., Hillman D. Daytime Predictors of Sleep Hypoventilation in Duchenne Muscular Dystrophy. Am J Respir Crit Care Med 2000; 161: 166-170.
14. Ragette R., Mellies U., Schwake C., Voit T., Teschler H. Patterns and predictors of sleep disordered breathing in primary myopathies. Thorax 2002;57:724-728.

15. Zenteno D et al. Guías de rehabilitación respiratoria en niños con enfermedades respiratorias crónicas: actualización 2016. Neumol Pediatr 2016; 11: 114 - 131.

16. Szeinberg A, Marcotte JE, Roizin $\mathrm{H}$, et al. Normal values of maximal inspiratory and expiratory pressures with a portable apparatus in children, adolescents, and young adults. Pediatr Pulmonol 1987; 3: 255-8.

17. Mellies U, Ragette R, Schwake C, Boehm H, Voit T, Teschler $\mathrm{H}$. Daytime predictors of sleep disordered breathing in children and adolescents with neuromuscular disorders. Neuromuscul Disord. $2003 ; 13: 123-8$

18. Redline S; Budhiraja R; Kapur V et al. Reliability and validity of respiratory event measurement and scoring. J Clin Sleep Med. 2007:3;169-200.

19. Simonds AK. Recent advances in respiratory care for neuromuscular disease. Chest 2006; 130: 1879-86.

20. Nozoe K, Moreira G, Tolino J, Pradella-Hallinan M, Tufik $S$, Andersen $M$. The sleep characteristics in symptomatic patients with Duchenne muscular dystrophy. Sleep Breath. 2015;19:1051-6.

21. Clinical Guidelines for the Manual Titration of Positive Airway Pressure in Patients with Obstructive Sleep Apnea. Positive Airway Pressure Titration Task Force of the American Academy of Sleep Medicine. J Clin Sleep Med. 2008 15; 4: 157-171.

22. Best clinical practices for the sleep center adjustment of noninvasive positive pressure ventilation (NPPV) in stable chronic alveolar hypoventilation syndromes. NPPV Titration Task Force of the American Academy of Sleep Medicine. J Clin Sleep Med. 2010 15;6:491-509.

23. Paiva R., Krivec U., Aubertin G., Cohen E., Clement A., Fauroux B. Carbon dioxide monitoring during long-term noninvasive respiratory support in children. Intensive Care Med. 2009;35:1068-74

24. Caldarelli V., Borel JC, Khirani S, Ramirez A, Cutrera R, Pepin $\mathrm{JL}$, Fauroux B. Polygraphic respiratory events during sleep with noninvasive ventilation in children: description, prevalence, and clinical consequences. Intensive Care Med. 2013;39:739-46.

25. Finder JD, Birnkrant D, Carl J, Farber HJ, Gozal D, lannaccone ST, Kovesi T, Kravitz RM, Panitch H, Schramm C, Schroth M, Sharma G, Sievers L, Silvestri JM, Sterni L; American Thoracic Society. Respiratory care of the patient with Duchenne muscular dystrophy: ATS consensus statement. Am J Respir Crit Care Med. 2004;170: 456-65.

26. SomnoNIV Group. Proposal for a systematic analysis of polygraphy or polysomnography for identifying and scoring abnormal events occurring during non-invasive ventilation. Thorax. 2012 ;67:546-52.

27. Fauroux B. et al. Noninvasive positive-pressure ventilation avoids recannulation and facilitates early weaning from tracheotomy in children. Pediatr Crit Care Med. 2010; 11:31-7. 